\title{
Bundesstatistikgesetz 2000 - Vorgaben, Motive, Ziele
}

\author{
Ewald Kutzenberger ${ }^{1}$ und Josef Richter ${ }^{2}$ \\ ${ }^{1}$ Amt der Oberösterreichischen Landesregierung, Linz \\ ${ }^{2}$ Wirtschaftskammer Österreich, Wien
}

\begin{abstract}
Zusammenfassung: Die Bestimmungen des Bundesstatistikgesetzes 2000 werden vor dem Hintergrund der vier zentralen politischen Ziele gesehen, denen das Gesetz verpflichtet ist: Erstens der Reorientierung des statistischen Systems, um die Unabhängigkeit der Statistik zu stärken und ihre Funktion als Öffentliches Gut zu sichern. Zweitens die Voraussetzungen für eine deutliche Verbesserung der Produktionsbedingungen für die amtliche Statistik zu schaffen. Drittens die Belastung von Bürgern und Betrieben merkbar $\mathrm{zu}$ reduzieren und viertens den Bedingungen des neuen Datenschutzrechts zu genügen. Wenn Defizite im Blick auf die Vision eines idealen statistischen Systems verbleiben, so sind sie das Resultat von Zielkonflikten, aber auch dadurch bedingt, daß eine Verwirklichung tiefe Eingriffe in bestehendes Recht notwendig gemacht hätte.
\end{abstract}

Die Umwandlung des Österreichischen Statistischen Zentralamts in eine Anstalt öffentlichen Rechts dient der besseren Umsetzbarkeit der inhaltlichen Vorgaben des Gesetzes. Das Gesetz räumt der Leitung der Anstalt mehr Flexibilität und Planbarkeit bei größerer Verantwortung für die Ergebnisse ein. Mit der Anstalt „Statistik Österreich“ wurden die Weichen zur Schaffung eines echten Kompetenzzentrums für qualitativ hochwertige, unabhängige und aktuelle statistische Daten gestellt. Die Bundesstatistik wird in die Lage versetzt, mit dem Bürger einen Pakt zu schließen, aus dem beide Partner Gewinn ziehen.

\section{Einleitung}

Ein Bundesgesetz ist Ausdruck des gestalterischen Willens des Gesetzgebers. In einem gewissen bescheidenem Maße trägt es auch die Handschrift jener, welchen die Redaktion des Textes anvertraut war. Wie jedes Gesetz kann auch das Bundesstatistikgesetz nicht losgelöst vom historischen Kontext gesehen werden, vor dessen Hintergrund es initiiert wurde und vor dem es entstanden ist.

Die Beurteilung wird wesentlich davon abhängen, wieweit sich der Beurteiler mit den vorgegebenen Zielen identifizieren kann. Sie wird somit auch davon bestimmt werden, wieweit die eigenen a-priori-Vorstellungen umgesetzt wurden.

Ziel dieses Beitrags ist es nicht, eine Wertung des Gesetzes zu wagen. Ziel ist es auch nicht, die auslösenden Faktoren des neuen Gesetzes zu beschreiben oder eine Chronologie der Gesetzeswerdung anzubieten. Sehr wohl aber soll versucht werden, wichtige Ausgangspunkte und Vorgaben des Gesetzes darzustellen und auf die Motive und Ziele „hinter den Bestimmungen“ einzugehen. Vollständigkeit in der Darstellung wird nicht angestrebt; sie zu erreichen wäre auch kaum möglich. 
Da mehrere politische Ziele vorgegeben waren, die bei der Regelung einzelner Aspekte auch zueinander in Widerspruch standen, spiegeln einzelne Bestimmungen auch immer wieder das Ergebnis einer Güterabwägung, das Resultat einer Rangordnung der Ziele wieder.

Das Gesetz und seine Bestimmungen sind nicht zuletzt auch vor dem internationalen Hintergrund zu sehen: In vielen Staaten Europas ringt man um eine neue Definition der Ziele der Amtlichen Statistik, ringt man um eine neue Positionierung der Amtlichen Statistik im gesellschaftlichen System. Zwei Gründe zwingen zum Überdenken. Einerseits ist es der gewaltige Informationsbedarf der Regierungen, insbesondere auch jener der Europäischen Kommission. Andererseits ist es das neue Selbstverständnis der Bürger, die sich immer mehr wehren, zum Objekt einer einseitigen und überbordend angeordneten Informationsbeschaffung zu werden. Der Bürger erkennt vielfach den Zweck der Erhebungen nicht und sieht sich hilflos einer Flut von Befragungen ausgesetzt.

Aber auch die geänderten Möglichkeiten der Datenverwaltung und Datenverarbeitung machen ein Überdenken erforderlich. Die Statistikgesetze stammen in der Regel aus einer Zeit, in der EDV noch keine, oder nur eine völlig untergeordnete Rolle gespielt hat. Durch die heutigen Möglichkeiten, EDV bei der Datenbeschaffung und der Datenspeicherung auch in der Verwaltung einzusetzen, ergeben sich eine Reihe von Fragen, wie die Amtliche Statistik diesen Datenfundus nutzen kann, um einerseits den Datenschutz voll zu wahren und andererseits aber die Gesellschaft zu entlasten, das heißt Daten nicht zu erheben, welche die Verwaltung ohnedies schon hat.

Aus diesem Problemkreis heraus wurde durch eine Reihe von politischen Vorgaben eine neue Orientierung initiiert, wurden der Bundesstatistik neue Ziele gesetzt, denen das Bundesstatistikgesetz 2000 gerecht zu werden versucht. Das Gesetz will die Voraussetzungen dafür schaffen, einen neuen Pakt mit dem Bürger schließen zu können: Dieser gibt der Bundesstatistik Informationen, die sie sonst nirgends beschaffen kann. Als Gegenleistung ermöglicht ihm die Statistik den umfassenden und fairen Zugang zu allen Ergebnissen. Die Bundesstatistik soll zudem nicht hoheitlich anordnen, sie soll vielmehr eine Dienstleistung für den Bürger erbringen. Der Bürger hat ein Recht auf Statistische Informationen, er hat ein Recht auf einen objektiven Befund über die Gesellschaft.

\section{Politische Vorgaben}

Der Bericht des Verfassungsausschusses über die Regierungsvorlage nennt die politischen Zielsetzungen des Gesetzes:

1. Gesetzliche Festlegung der Voraussetzungen, bei deren Vorliegen die Erstellung von Statistiken und die Durchführung von statistischen Erhebungen mittels Verordnung durchgeführt werden kann.

2. Genauere Festlegung der Inhalte, die in einer Verordnung, mit der die statistischen Erhebungen angeordnet werden, enthalten sein müssen.

3. Einschränkung der Möglichkeit der Erhebung von personenbezogenen Daten sowie grundsätzliche Verpflichtung zur Löschung des Personenbezuges, sobald die Statistik, für die die Erhebung erfolgt ist, erstellt worden ist. 
4. Verpflichtung zur Minimierung der Belastung der Respondenten bei der Erstellung der statistischen Unterlagen.

5. Schaffung einer gesetzlichen Grundlage für die Erstellung und Fortführung von Respondentenregistern, die für die Auswahl von Auskunftspflichtigen bei der Anordnung von statistischen Erhebungen von wesentlicher Bedeutung sind.

6. Schaffung einer gesetzlichen Grundlage für die Erhebung von Daten bei öffentlich zugänglichen Registern und bei Verwaltungsdienststellen.

7. Verschärfung der Regelungen über das Statistikgeheimnis.

8. Umfangreiche Verpflichtung zur Veröffentlichung von statistischen Ergebnissen, um der breiten Öffentlichkeit im Interesse einer verstärkten Transparenz Zugang zu den Statistiken zu verschaffen.

9. Neuregelung der Zuständigkeit für die Durchführung von Verwaltungsstrafverfahren bei Nichterfüllung der Auskunftspflicht.

10. Umwandlung des Österreichischen Statistischen Zentralamtes in eine Bundesanstalt öffentlichen Rechts und Errichtung eines Wirtschaftsrates, der die wirtschaftliche Gestion der Bundesanstalt überwacht.

11. Festlegung der Grundsätze, nach denen die Bundesanstalt die Aufgaben wahrzunehmen hat.

12. Schaffung eines mit externen Fachleuten besetzten Statistikrates bei der Bundesanstalt zur Überprüfung der festgelegten Grundsätze bei der Erstellung der Statistiken sowie zur Beratung in Angelegenheiten der Koordination der Bundesstatistik, vor allem in Richtung einer Entlastung der Respondenten und der Ressourcen.

13. Verpflichtung der Bundesanstalt, die Veröffentlichungen der Statistiken auch über das Internet unentgeltlich bereitzustellen.

14. Regelung des Zugangs der Wissenschaft zu den Statistikdaten der Bundesanstalt unter Sicherstellung des Datenschutzes.

15. Schaffung einer gesetzlichen Grundlage für die Erfüllung der Auskunftspflicht durch Respondenten auf elektronischem Wege.

Darüber hinaus sollen EU-Rechtsnormen, die für den Bereich der Statistik und den Bereich des Datenschutzes gelten, bei der Durchführung von statistischen Erhebungen mitberücksichtigt werden.

Versucht man, diese Ziele zusammenzufassen und zu bündeln, lassen sich - vereinfachend gesprochen - vier zentrale Anliegen erkennen:

1. Reorientierung des statistischen Systems; Stärkung der Unabhängigkeit der Statistik; Statistik ist als Öffentliches Gut zu sehen; 
2. Effizienz der Statistikerstellung anheben, die „Produktionsbedingungen“der Amtlichen Statistik mit dem Ziel verbessern, Rationalisierungspotentiale nutzen zu können, um zumindest mittelfristig eine Budgetentlastung zu erreichen; Schaffung einer neuen Organisationsform;

3. Belastung der Bürger und der Firmen vermindern;

4. Bedingungen des neuen Datenschutzrechts genügen.

Der Erreichung dieser Ziele sollen einerseits die Bestimmungen der ersten 32 Paragraphen dienen, die als der „Statistikteil“ des Gesetzes angesprochen werden können. Paragraph 22 und die Paragraphen 33 bis 62 sind der Errichtung der Bundesanstalt ,Statistik Österreich“ gewidmet. ${ }^{1}$ Mit der Ausgliederung des Österreichischen Statistischen Zentralamts und der Schaffung einer neuen Organisationsform sollen vor allem die Bedingungen für die Umsetzung der im Statistikteil niedergelegten Grundsätze verbessert werden.

\section{Umsetzung der Vorgaben im ,Statistikteil“ des Geset- zes}

\subsection{Reorientierung des statistischen Systems}

\subsubsection{Funktion der Bundesstatistik}

In $\S 1$ ist erstmals eine Zielbestimmung enthalten die festlegt, worüber die Bundesstatistik Informationen sammeln und für wen sie diese zur Verfügung zu stellen hat. Demnach soll das aufzubauende Informationssystem Daten über die wirtschaftlichen, demografischen, sozialen, ökologischen und kulturellen Gegebenheiten in Österreich enthalten. Die Bundesstatistik hat einen objektiven Befund über unsere Gesellschaft zu erstellen und die Ergebnisse gleichermaßen den Bundesorganen - zur Planung, Entscheidungsvorbereitung und Kontrolle von Maßnahmen - und der Wissenschaft, der Wirtschaft und der Öffentlichkeit bereitzustellen.

Damit die Bundesstatistik diese Daten (Informationen) liefern kann, darf sie Statistiken aller Art erstellen ( $(2)$. Eingeschlossen sind die damit zusammenhängenden Analysen, die Berechnung von Indizes, von Indikatoren, die Erstellung von Prognosen und der Einsatz statistischer Modelle. Mit dieser Festlegung ist gemeint, daß die Statistik nicht einfach Daten sammeln soll, sondern sie über Analysen aufwerten muß. Den Daten ist - weit über das Darstellen einer Zahl in einer Tabelle - eine zusätzliche Qualität mitzugeben. Der Modellbau und damit das Zusammenspiel von vielen Merkmalen aus verschiedenen Erhebungen in kohärenten Systemen zur Simulation von Zuständen, über die wir sonst nichts wissen, ist die erstrebenswerte Zukunft der Amtlichen Statistik: Das Schaffen von Entscheidungsgrundlagen auf höchster Qualitätsebene.

\footnotetext{
${ }^{1}$ Wenn nicht ausdrücklich anders vermerkt, beziehen sich alle Paragraphen auf die Bestimmungen des Bundesstatistikgesetzes 2000.
} 
Die im $\S 3$ festgehaltene Definition zum Begriff Statistik unterstreicht dies noch einmal: Statistik ist die quantitative Beschreibung und Beurteilung von Massenerscheinungen.

Beschreibung spricht die klassische Form der Statistik an, das Erstellen und Beschreiben von Tabellen.

Beurteilung meint, daß die Information zusätzlich durch eine Qualität angereichert wird. Vergleiche über die Zeit, Vergleiche mit anderen Regionen, die statistische Beurteilung der Signifikanz gehen über die reine Beschreibung einer Zahl hinaus. Eine äußerst wichtige Funktion, die der Statistik erst ermöglicht, hochwertige Information zu liefern. Nicht nur das Schreiben von Tabellen, sondern auch die statistisch-qualitative Beurteilung der Information muß möglich sein. Es sollte per Definition eine Verpflichtung der Statistiker sein, bei ihrer Arbeit weit über das reine Datensammeln hinauszugehen.

Mit Massenerscheinungen wird dokumentiert, daß die Statistik nicht am identifizierbaren Individuum interessiert ist. Das anonyme Einzeldatum ist notwendig zur Erreichung eines Ergebnisses über eine Masse. Der überaus sorgsame Umgang mit Informationen zum Schutze des Einzelnen ist Verpflichtung der Statistik. Es geht um den Aufbau eines nicht-personenbezogenen Informationssystems.

Die Definition von Statistik am Beginn des Gesetzes legt fest, was Statistik zu sein hat und was sie nicht ist. Im $\S 14$ werden alle Organe der Bundesstatistik auf klare Ziele und Grundsätze festgelegt. Mit diesen Vorgaben wird der Öffentlichkeit signalisiert, welchen Grundsätzen die Bundesstatistiker verpflichtet sind. Damit soll Verständnis in der Öffentlichkeit geweckt und das Ansehen der Statistik gefördert werden.

Die in $\S 14$ normierten Grundprinzipien sind Objektivität, Zuverlässigkeit, Erheblichkeit, Kostenwirksamkeit, Transparenz und möglichst hohe Kohärenz. Diese Prinzipien entsprechen den in der EU-Verordnung über Gemeinschaftsstatistiken genannten Grundsätzen.

An die Bundesanstalt „Statistik Österreich“ werden darüber hinaus in $\S 24$ besondere Anforderungen bei der Wahrnehmung ihrer Aufgaben gestellt, welche die Pflicht zur Professionalität und Qualität dokumentieren:

- Objektivität und Unparteilichkeit

- Einsatz von Methoden und Verfahren nach dem letzten Standard

- Pflicht zur ständigen Qualitätsverbesserung

- Streben nach höchster Aktualität der Statistiken

- Minimierung der Belastung und ausreichende Information der Auskunftspflichtigen

- Wahrung der Grundsätze der Veröffentlichung und der

- Vertraulichkeit von personenbezogenen Daten.

Für die Bundesanstalt „Statistik Österreich“ wird in $\S 22$ besonders deutlich hervorgehoben, daß ihr die Erbringung von Dienstleistungen wissenschaftlichen Charakters im öffentlichen Interesse obliegt. Sie ist nicht auf Gewinn orientiert. Damit ist klargestellt, daß es sich bei einem Ergebnis der Bundesstatistik nicht um ein Produkt handelt, das man 
nur aus wirtschaftlichen Gesichtspunkten allein bewerten kann. Es geht um die Beurteilung der Verhältnismäßigkeit des Aufwandes zu einer dem Problem adäquaten Qualität. Die Bundesanstalt „Statistik Österreich“ ist auch ausdrücklich beauftragt, auf die adäquate Verwendung und Interpretation von veröffentlichten Statistiken hinzuwirken ( $§ 29$ ).

Die Einhaltung aller dieser Grundsätze hat als Aufsichtsorgan der „Statistikrat“ zu kontrollieren (s. dazu Pkt. 4.2).

\subsubsection{Geänderte Publikationspolitik}

In der Publikationspolitik erfolgt eine echte Reorientierung. Statistik wird nicht mehr primär als Dienerin der Verwaltung - in letzter Zeit vor allem der EU-Kommission sondern als die Erbringung einer Dienstleistung wissenschaftlichen Charakters für die gesamte Öffentlichkeit, Politik, Wirtschaft, Wissenschaft, für alle Bürger angesehen. Die Publikationsbestimmungen entsprechen der in Punkt 1 der „Fundamental Principles“ der Economic Commission for Europe niedergelegten Forderung „Official statistics ... are to be made available on an impartial basis by official statistical agencies to honour citizens' entitlement to public information."

Dem Ziel eines fairen Datenzuganges dient, daß Statistiken grundsätzlich und unverzüglich zu veröffentlichen sind. Ausnahmen von dieser generellen Pflicht werden nur in eng begrenzten Fällen eingeräumt. Es wird das Verbot der Bevorzugung politischer Entscheidungsträger beim Informationszugang ebenso normiert wie die Pflicht zur Gleichstellung österreichischer Interessenten mit EU Stellen (§ 30). Die Amtliche Statistik wird die ihr zukommende Funktion als konstitutives Element eines demokratischen Staats wahrnehmen können und müssen.

Die zur Interpretation unverzichtbaren Konzepte, Definitionen und Erläuterungen sind zu publizieren ( $(19(1))$. Auch klassifikatorische Zuordnungen sind jedem bekanntzugeben, der ein berechtigtes Interesse an dieser Information glaubhaft machen kann (§ 21(8)).

Damit das Recht des Bürgers auf das öffentliche Gut Statistik nicht nur formal, sondern auch materiell gewährleistet ist, wurde zu den schon bisher bestehenden Publikationsverpflichtungen die Pflicht zur umfassenden und kostenlosen Publikation im INTERNET (§ 30(1)) aufgenommen. Die kostenlose Bereitstellung der Statistiken im INTERNET darf sich nicht auf wenige Hauptergebnisse beschränken. In den Erläuterungen zum Gesetz ist festgehalten, daß - nach einer Übergangszeit - jener Publikationsumfang erreicht werden muß, wie er derzeit in der Schriftenreihe „Beiträge zur österreichischen Statistik" gegeben ist.

Dem Charakter statistischer Ergebnisse als Öffentliches Gut wird auch gerecht, daß an Kosten für spezifische „maßgeschneiderte“ Auswertungen nur aufgelaufene Zusatzkosten verrechnet werden dürfen.

In all diesen Aspekten können die Publikationsvorschriften auch anderen europäischen Staaten als Vorbild dienen. Sie kontrastieren sehr positiv zur Publikationspolitik von EUROSTAT. Entgegen zahlreicher Lippenbekenntnisse ist die Kommission beim Zugang zum Datenfundus deutlich privilegiert, die Nutzung des Datenangebots durch Außenstehende ist - wenn überhaupt - nur zu prohibitiv hohen Kosten möglich. 


\subsubsection{Unabhängigkeit in fachlich methodischen Fragen}

Nach $\S 38$ ist der fachliche Leiter der Bundesanstalt „Statistik Österreich“ bei der Erfüllung seiner Aufgaben in allen wissenschaftlich methodischen Fragen nicht an Weisungen einer politischen Instanz gebunden.

Diese Autonomie im fachlichen Bereich stellt für Österreich ein Novum dar. Wäre das Statistische Zentralamt eine Bundessdienststelle geblieben, hätte es einer Verfassungsbestimmung bedurft, um diese für die Gewinnung des Vertrauens der Öffentlichkeit so unverzichtbare Weisungsfreiheit verankern zu können.

Eingeschränkt wird die Autonomie nur durch einige Festlegungen im Gesetz mit dem Ziel der Sparsamkeit und der Respondentenschonung selbst: Wenn immer möglich, ist der Weg der Sekundärstatistik zu wählen, Stichprobenerhebungen ist der Vorzug gegenüber Vollerhebungen zu geben.

\subsection{Verbesserung der Produktionsbedingungen der Amtlichen Stati- stik, Nutzung von Möglichkeiten der Einsparung}

Eine wesentliche Voraussetzung dafür, administrative Daten sinnvoll nutzen zu können ist, daß gleiche statistische Einheiten gleich klassifiziert sind. Das Gesetz sieht daher vor, daß wer immer auf Grund gesetzlicher Regelungen nach statistischen Systematiken Klassifikationen vorzunehmen hat, sich der Zuordnungen zu bedienen hat, welche die Bundesanstalt „Statistik Österreich“ vorgenommen hat (§ 21 (1)). Die Amtliche Statistik wird somit mit einem Klassifikationsmonopol ausgestattet.

Die Bundesanstalt ist von Amts wegen zur klassifikatorischen Zuordnung und zur adäquaten Wartung dieser Zuordnungen verpflichtet ( $§ 21$ (2)). Die Zuordnungen sind allen Einrichtungen, die mit diesen Zuordnungen zu operieren haben, schriftlich und kostenlos zu übermitteln, die Informationsübermittlung kann auf elektronischem Weg erfolgen $(\S 21(3))$.

Wegen der vielen möglichen Rechtsfolgen, die nach europäischem Recht an klassifikatorische Zuordnungen (vor allem was die Aktivitätszuordnungen von Einheiten betrifft) geknüpft sind, enthält das Gesetz auch eine Reihe von Verfahrensbestimmungen, welche die Behandlung von Einsprüchen gegen vorgenommene Zuordnungen regeln.

Einem zentralen Register kommt für die Nutzung administrativer Daten eine strategische Bedeutung zu. Das Gesetz sieht daher für die Bundesanstalt „Statistik Österreich“ die Führung eines zentralen Registers vor und regelt die Verwendung auch personenbezogener Daten für die Registerführung ( $§ 25)$.

Über juristische Personen, Einrichtungen, Unternehmen und ihre Betriebe und Arbeitsstätten sowie über Arbeitsgemeinschaften und Forschungsstätten dürfen personenbezogene Register mit folgenden Merkmalen als regelmäßig ergänzte Datensammlungen geführt werden:

1. Identifikationsmerkmale;

2. Adreßmerkmale;

3. Systematikmerkmale; 
4. Referenzmerkmale;

5. Versand- und Auskunftsmerkmale;

6. Datenquellenmerkmale.

Die statistische Auswertung der Registerinformation ist auf diese Merkmale beschränkt.

Zur Erstellung und Wartung dürfen auch personenbezogene Daten aus durchgeführten statistischen Erhebungen sowie Daten aus öffentlichen Registern und Verwaltungsdaten herangezogen werden. Aus datenschutzrechtlichen Überlegungen ist das Register im wesentlichen auf den Bereich der Wirtschaftsstatistiken in weiterem Sinne eingeschränkt. Sonstige einzelfall-, aber nicht personenbezogene Register, wie etwa Gebäuderegister, dürfen aber ebenfalls geführt werden ( $(23)$.

Was den Zugang zu administrativen Daten, die Möglichkeit des Registerabgleichs betrifft, schafft das Gesetz die erforderlichen Grundlagen, um die Produktionsbedingungen der Amtlichen Statistik zu verbessern. Nach einer sehr arbeitsintensiven Umstellungsphase und nach massiven Investitionen in die Ausstattung - sowohl materiell als auch in Humankapital - müßte eine spürbare Entlastung möglich sein.

Das Potential der für statistische Zwecke überhaupt nutzbaren administrativen Daten wird durch das Gesetz nicht vergrößert. Dieses Potential ist im Vergleich zu anderen europäischen Staaten relativ gering, da z.B. in Österreich umfangreiche Meldungen an die Finanzverwaltung fehlen, die in anderen Staaten die Grundlage für die Substitution wirtschaftsstatistischer Primärerhebungen schaffen.

Neben der Öffnung des Zugangs zu administrativen Daten erfüllt das Gesetz noch einen anderen wesentlichen Wunsch der Amtlichen Statistik. Ab 2000 wird es möglich sein, die finanziellen Mittel, die von internationalen Organisationen - wie etwa von der Europäischen Kommission - für statistische Projekte zur Verfügung gestellt werden, unmittelbar der Amtlichen Statistik zufließen zu lassen.

\subsection{Belastung der Bürger und der Firmen vermindern}

Diesem Ziel dienen eine Reihe von Bestimmungen. Die wichtigsten sind:

\subsubsection{Nutzung der verfügbaren administrativen Daten}

Um den bürokratischen Aufwand der Firmen zu reduzieren, sind zuerst alle vorhandenen administrativen Daten zu nutzen, bevor Direktbefragungen durchgeführt werden dürfen ( $\S$ 6(3)). Mit der Möglichkeit, bereits verfügbare Information aus der Verwaltung verwenden zu können, wird eine ganz zentrale Forderung der Wirtschaft, aber auch der Bundesstatistik, erfüllt.

Aus dieser Priorität für die Nutzung bereits verfügbarer Information resultieren umfangreiche Mitwirkungspflichten von Verwaltungsstellen. Diese Pflichten sind in $\S 10$ zusammengefaßt: Beim Heranziehen von Register, Verwaltungs- und Statistikdaten haben die Stellen, die öffentliche Register führen, sowie die Inhaber von Verwaltungsdaten und Statistikdaten die Daten zu übermitteln, soweit dies in einer Anordnung gemäß $\S 4$ vorgesehen ist oder Daten für die Register gemäß $\S 25$ benötigt werden. Im Zuge der 
Übermittlung sind dem Organ der Bundesstatistik auf dessen Verlangen auch die erforderlichen Metadaten, wie Merkmalsdefinitionen, auf welche Art die betreffenden Daten angefallen sind und welche Berechnungsmethoden angewandt wurden, bereitzustellen. Die Übermittlung hat unentgeltlich und auf elektronisch lesbaren Datenträgern zu erfolgen, wenn die Daten in elektronisch lesbarer Form vorhanden sind.

Bei der Einrichtung und Änderung von öffentlichen Registern, die Daten in elektronisch lesbarer Form enthalten oder enthalten werden, die für eine statistische Erhebung oder für die Ergänzung der Register gemäß $\S 25$ relevant sein können, ist zudem auch auf die Erfordernisse der Statistik bedacht zu nehmen. Inhaber von Verwaltungsdaten sind verpflichtet, auf Verlangen Auskunft über das Vorhandensein von Verwaltungsdaten, die für eine statistische Erhebung oder für die Ergänzung der Register gemäß $\S 25$ relevant sein können, zu geben. Falls diese Information in elektronisch lesbarer Form in einer Datei gespeichert vorliegt, ist darüber hinaus über den Aufbau und die Struktur der Dateien Information bereitzustellen.

Mit all diesen Bestimmungen werden Verwaltungsstellen neue Aufgaben zugewiesen, es wird in etablierte Traditionen eingegriffen. Da manche dieser Aufgaben nicht im unmittelbaren Eigeninteresse der Verwaltungsstellen liegen, kann Begeisterung für die neuen Mitwirkungspflichten kaum vorausgesetzt werden. Die Gewinnung einer aktiven Kooperationsbereitschaft der „Hüter“ der administrativen Daten wird sicher zu den vorrangigen Zielen der Leitung der Anstalt gehören müssen. Ein „Gesamtdenken“ der öffentlichen Verwaltung bei der Nutzung der vorhandenen Informationen sollte das Ziel sein.

\subsubsection{Praxisnahe Erhebungsunterlagen}

Bei der Gestaltung der Meldeunterlagen ist auf die möglichst geringe Belastung und auf die Besonderheiten der zu Befragenden (Branche, Betriebsgröße) Rücksicht zu nehmen ( $\S$ 14(2)). Als Vorbild kann „Statistics Canada“ dienen. Dieses Amt bietet bei Wirtschaftsbefragungen 270 verschiedene Fragebögen an, abgestimmt auf Branche und Betriebsgröße.

\subsubsection{Freiwilligkeit statt Zwang}

Wenn immer möglich, sind Erhebungen auf der Grundlage freiwilliger Mitarbeit durchzuführen $(\S 6(2))$.

\subsubsection{Stichproben anstatt Vollerhebungen}

Grundsätzlich ist Stichprobenerhebungen der Vorzug vor Vollerhebungen zu geben $(\S 7$ (1)). Bei laufend durchzuführenden Erhebungen ist ein Austausch der Auskunftspflichtigen in der Stichprobe anzustreben $(\S 7(3))$.

\subsubsection{Pflicht zur kostenlosen Bereitstellung ,elektronischer Fragebögen“6}

Die Möglichkeiten der modernen Informationstechnologie sollen verstärkt genutzt werden. Betrieben ist die Möglichkeit der elektronischen Meldungen zu eröffnen, ,elektronische Fragebögen“ sind in Zukunft kostenlos anzubieten ( $§ 28$ ). Diese Bestimmung entla- 
stet die Betriebe, müßte gleichzeitig aber auch auf mittlere Frist zu Kosteneinsparungen im Produktionsprozeß der Anstalt führen und vor allem der höheren Aktualität der Daten förderlich sein.

\subsection{Bedingungen des neuen Datenschutzrechts genügen}

Statistik zielt auf komprimierte, entindividualisierte Information. Dennoch tangiert Statistik häufig das Recht auf informationelle Selbstbestimmung. Dies ist in besonderem Maße der Fall, wenn bei statistischen Erhebungen Meldepflicht besteht oder wenn Daten, welche für andere Zwecke gewonnen wurden, statistisch aufgearbeitet werden.

Das gleichzeitig mit dem Bundesstatistikgesetz 2000 beschlossene Datenschutzgesetz 2000 enthält in seinem $\S 46$ Sonderregelungen für die Datenverwendung durch die wissenschaftliche Forschung und für statistische Zwecke:

Für Zwecke wissenschaftlicher oder statistischer Untersuchungen, die keine personenbezogenen Ergebnisse zum Ziel haben, dürfen generell öffentlich zugängliche Daten, indirekt personenbezogene Daten oder Daten, die der Auftraggeber für andere Zwecke zulässigerweise ermittelt hat, verwendet werden. Diese Bedingungen treffen für die meisten statistischen Arbeiten nicht zu.

Andere Daten dürfen für wissenschaftliche oder statistische Untersuchungen nur verwendet werden, wenn eine der drei folgenden Bedingungen erfüllt ist:

- wenn eine besondere gesetzliche Vorschrift vorliegt,

- mit Zustimmung der Betroffenen oder

- mit Genehmigung der Datenschutzkommission.

Eine der zentralen Funktionen des Bundesstatistikgesetzes 2000 ist es, die besonderen gesetzlichen Vorschriften des $\S 46$ (2) Z 1. des Datenschutzgesetzes 2000 bereitzustellen. Das Bundesstatistikgesetz erfüllt dabei die Funktion eines Rahmengesetzes, das im $\S 5$ die Zulässigkeit der Anordnung personenbezogener Erhebungen durch Verordnungen im Detail regelt.

Dem Ziel eines sehr hohen Niveaus des Datenschutzes dient auch, daß - wenn immer sachlich möglich - personenbezogene Daten zu anonymisieren sind (§ 15). In diesem Zusammenhang ist auch für die Wissenschaft keine Ausnahme vorgesehen. Das Statistikgeheimnis wird weiter verschärft. Mit Aufgaben der Bundesstatistik betraute Personen sind über alle personenbezogenen Daten zur strikten Verschwiegenheit verpflichtet. Verstöße sind nach dem Strafgesetzbuch zu ahnden (§ 17).

Vor allem wird sehr deutlich festgehalten, daß zu statistischen Zwecken erhobene Daten nicht zu Verwaltungszwecken eingesetzt oder sonst weitergegeben werden dürfen. Diesem strikten ,Einbahnstraßenprinzip“ entspricht, daß personenbezogene Daten an Dritte nur übermitteln werden dürfen, wenn besondere gesetzliche Regelungen vorliegen oder wenn der Betroffene ausdrücklich und unmißverständlich der Übermittlung zugestimmt hat $(\S 17(2))$.

Die Veröffentlichung von Statistiken hat so zu erfolgen, daß ein Rückschluß auf Angaben über bestimmte oder bestimmbare Betroffene ausgeschlossen werden kann. Die 
Publikation darf jedoch erfolgen, wenn eine vorherige und ausdrückliche schriftlicher Zustimmung des Betroffenen vorliegt ( $§ 19(2)$ ).

In Hinblick auf die Ziele optimale Produktionsbedingungen, Budgetentlastung und größtmögliche Schonung der Respondenten erscheinen die Vorgaben des Datenschutzes restriktiv und behindernd. Vielen erscheint auch der in der Verrechtlichung zu sehende Preis unangemessen hoch. Der Qualität der Statistik hingegen kann ein sehr hohes Datenschutzniveau nur förderlich sein. Respondenten sind nur zu wahrheitsgemäßen Angaben zu motivieren, wenn sie sicher sein können, daß ihre Angaben streng vertraulich behandelt werden und nie für administrative Zwecke gegen sie selbst eingesetzt werden können. Das Vertrauen der Respondenten ist für die Statistik ein so hohes Gut wie das Vertrauen in die Wahrung des Bankgeheimnisses für ein Geldinstitut.

\subsection{Unerfüllte Wünsche}

Da das Bundesstatistikgesetz mehreren Zielen entsprechen will, waren Zielkonflikte unvermeidbar. Die vollständige Umsetzung aller ,statistischen Anliegen“ hätte zudem massiv in andere Rechtsvorschriften eingreifen müssen, in zahlreichen Fällen wäre eine Änderung der Bundesverfassung unumgänglich geworden.

Von der Warte einer isoliert gesehenen Vision eines idealen statistischen Systems aus gesehen, entstanden Defizite. Auch jeder, der mit der impliziten Hierarchie der Ziele hinter den Bestimmungen nicht übereinstimmt, wird unerfüllte Wünsche konstatieren. Die wichtigsten dieser Wünsche sollen kurz behandelt werden.

\subsubsection{Umfassende Regelung für alle durch Gesetze angeordnete Statistiken}

Die ursprüngliche Absicht, alle auf der Grundlage von Bundesgesetzen erfolgenden statistischen Aktivitäten im Bundesstatistikgesetz zu regeln, mußte fallengelassen werden. Ihre Verwirklichung hätte tiefe Eingriffe in bestehendes Recht erforderlich gemacht, die Frage der Autonomie der Informationsbeschaffung von Institutionen wie der Oesterreichischen Nationalbank, der gesetzlichen Interessenvertretungen und ähnlicher Einrichtungen hätte überdacht werden müssen. Im Interesse einer raschen Verabschiedung eines Bundesstatistikgesetzes wurde auf die Diskussion dieser Grundsatzfragen verzichtet. Die ursprüngliche Intention einer umfassenden Regelung hat aber in der Struktur des Gesetzes deutliche Spuren hinterlassen.

Das 1. Hauptstück enthält nicht nur Begriffsbestimmungen, sondern regelt alle grundsätzlichen Aspekte, die Anordnung von Statistiken, die Mitwirkungspflichten und die Pflichten aller Organe der Bundesstatistik. Erst das 2. Hauptstück ist dann dem zentralen Organ der Bundesstatistik, der neu zu errichtenden Bundesanstalt „Statistik Österreich“, gewidmet.

Das Gesetz beschränkt sich darauf, die Bundesstatistik zu regeln. Die Bundesstatistik umfaßt nach $\S 2$ die Erstellung von Statistiken aller Art, die über die Interessen eines einzelnen Landes hinausgehen. Organe der Bundesstatistik sind die Bundesanstalt „Statistik Österreich“ und jene Bundesdienststellen, die auf der Grundlage eines innerstaatlich unmittelbar wirksamen Rechtsakts oder durch Bundesgesetz berufen sind, Daten zu erheben und Statistiken zu erstellen ( $(3 \mathrm{Z}$ 19.). Die Beschränkung auf „Bundesdienststellen“ 
schließt die Körperschaften öffentlichen Rechts damit ebenso aus wie die Oesterreichische Nationalbank. Durch das Bundesstatistikgesetz wird die Statistikkompetenz und die Rechtssituation dieser Institutionen hinsichtlich der Erstellung von Statistiken nicht verändert.

Da die Bundesanstalt nach $\S 23$ (1) die gemäß $\S 4$ des Bundesstatistikgesetzes angeordneten Erhebungen durchzuführen hat, beziehen sich die allgemeinen wie die besonderen Regelungen des Gesetzes vor allem auf die Tätigkeit der Bundesanstalt „Statistik Österreich“. Als andere Organe der Bundesstatistik kommen nur Bundesministerien in Frage. Sie können im Rahmen ihres Wirkungsbereichs Statistiken erstellen, soweit das Erhebungsmaterial im Rahmen des Geschäftsbetriebs anfällt und soweit die Ergebnisse ausschließlich für den Gebrauch der betreffenden Bundesministerien bestimmt sind ( $\S$ 20).

\subsubsection{Umfassende Koordinierungskompetenz für die Anstalt}

Vor dem Hintergrund der psychologisch und finanziell ungemein belastenden Doppelund Dreifacherhebungen von gleichen oder sehr ähnlichen Daten durch verschiedenste Institutionen, wäre in Hinblick auf das Ziel der Respondentenentlastung eine zentrale Koordinierungskompetenz sehr erstrebenswert. Auch sie hätte aber in die Autonomie potentieller Ersteller oder Auftraggeber, wie der Notenbank, der Hochschulen, der gesetzlichen Interessensvertreter eingreifen müssen.

Wie schwierig eine solche Beschränkung oder auch nur Abgrenzung zu erreichen ist, zeigten die Verhandlungen zur Verordnung des Rates über die Erfassung statistischer Daten durch die Europäische Zentralbank (EZB) zur sogenannten „EZB StatistikVerordnung“. Auch der EZB wurde eine nahezu uneingeschränkte Statistikkompetenz als für ihre Unabhängigkeit konstitutiv zugestanden. Eine Koordinierung zur Vermeidung von Doppelgleisigkeiten obliegt dem ,Ausschuß für Währungs-, Finanz- und Zahlungsbilanzstatistik“. In einem Annex zu seiner Geschäftsordnung legte dieser Ausschuß ausschließliche Kompetenzen für EUROSTAT und die EZB fest, primäre Kompetenzen für jeweils eine der Institutionen und schließlich Arbeitsgebiete, die in die Interessensphäre beider Institutionen fallen.

Ein dem „Ausschuß für Währungs-, Finanz- und Zahlungsbilanzstatistik“ korrespondierendes Organ für die Koordinierung zwischen Bundesanstalt und Oesterreichischer Nationalbank sieht das Bundesstatistikgesetz nicht vor. Die Zusammensetzung des Statistikrates, in den viele der wesentlichen Statistik betreibenden Institutionen fachkundige Vertreter zu entsenden haben, läßt aber hoffen, daß eine weitgehende Koordinierung und nicht nur mit der Nationalbank - durch „Gentlemen Agreement“ auf mittlere Sicht möglich sein wird.

\subsubsection{Autonomie in der Programmgestaltung}

In einem Vortrag über Anforderungen an ein modernes Statistikrecht vor der Statistischen Zentralkommission am 2. April 1998 in Wien hat Heinrich Brüngger die Regelung der Frage, wer über die statistischen Aktivitäten in einem Land zu entscheiden habe, als einen zentralen Punkt jedes Statistikgesetzes genannt. Dies gelte in besonderem Maße für alle Erhebungen, weil Erhebungen - im Gegensatz zu den anderen Tätigkeiten - zu einer 
zusätzlichen Belastung von zu befragenden Personen oder Unternehmen führen. „Ferner ist es wichtig, zwei Kategorien von Entscheidungen zu unterscheiden: Entscheide über das ,was', d.h. darüber, welche Informationsbedürfnisse als relevant für Gesellschaft und Staat zu gelten haben und wie innerhalb dieses Bereichs allenfalls die Prioritäten zu setzen sind, und Entscheide über das ,wie', d.h. darüber, mit welchen Methoden im einzelnen die benötigten Informationen erarbeitet und wie die Instrumente der Beschaffung der dazu nötigen Einzeldateneinzusetzen sind. Vom Gesichtspunkt der Belastung der Befragten abgesehen (insbesondere wenn sie eine Auskunftspflicht umfaßt), sollten Entscheide über das ,wie' in die fachliche Unabhängigkeit der Produzenten fallen und nicht von einer politisch tätigen Behörde gefällt werden. Es ist wichtig, daß klar gesagt wird, welches Organ für welche Entscheide bezüglich welcher Art von Statistiken zuständig ist." (Brüngger, 1998, S. 24)

Eine zentrale Forderung an ein Gesetz sei auch, daß es sich klar dazu äußert, wie die Spielregeln in diesen Entscheidungsprozessen sind. „Nur wenn diese für alle verbindlich und transparent sind, kann so etwas wie ein nationales Statistiksystem überhaupt geführt und bewußt gestaltet werden." (Brüngger, 1998, S. 24)

Diesen Kriterien entspricht das Bundesstatistikgesetz 2000. Die Abläufe werden offengelegt und es wird klargestellt, daß die letzte Entscheidung über das statistische Programm bei politisch Verantwortlichen liegt. Der fachliche Leiter der Anstalt ist nur in fachlich/methodischen Fragen weisungsfrei.

Eine „Programmautonomie“im Rahmen eines Globalbudgets ist für Österreich nicht vorgesehen. Wie Heinrich Brüngger in seinem Überblick über die legistischen Lösungsmodelle ausführte, ist eine solche Regelung vor allem in angelsächsischen Staaten und mit Abwandlungen - auch in skandinavischen Ländern anzutreffen. Eine Variante dieses Systems besteht darin, daß grundlegende Entscheide nicht vom Direktor, sondern von einer Art Verwaltungsrat des Statistischen Amtes ${ }^{2}$ gefällt werden. Viele Länder halten aber nach wie vor daran fest, daß es Sache einer politischen Behörde, z.B. der Regierung, sein muß, über das „was“ und die Verhältnismäßigkeit des ,wie“ zu entscheiden. Wichtig ist dann, daß diese Entscheide in einen Gesamtzusammenhang eingebettet sind und nicht einfach von Fall zu Fall getroffen werden. Ausdruck eines solchen Ansatzes sind die statistischen Mehrjahresprogramme, die aber mangels Verbindlichkeit (auch bezüglich des Ressourceneinsatzes) die ihnen zugedachte Rolle de facto oft nicht erfüllen können (s. Brüngger, 1998)

Das Bundesstatistikgesetz gibt der Leitung der Bundesanstalt vorerst eine respektable Gestaltungsmöglichkeit, wenn es regelt, daß sie das Jahresarbeitsprogramm und ein Vierjahresarbeitsprogramm zu erstellen und dem Statistikrat vorzulegen hat ( 339 (1)). Eingeschränkt wird diese Autonomie aber dadurch, daß die Programme die in $\S 23$ (1) angeführten Aufgaben zu enthalten haben (§ 39 (2)). Gemäß $\S 4$ (1) sind dies im wesentlichen alle durch einen innerstaatlich unmittelbar wirksamen internationalen Rechtsakt, durch Bundesgesetz oder durch eine Verordnung angeordnete Statistiken. Die Freiheit der Anstalt, daß auch sonstige Statistiken für öffentliche Stellen aufgrund vertraglicher Vereinbarungen erstellt werden dürfen $\S 23$ (2), wird ebenfalls erheblich eingeengt. Die Bundesanstalt darf solche Arbeiten nur durchführen, wenn die Erfüllung der gesetzlich

\footnotetext{
${ }^{2}$ Der in Österreich vorgesehene Statistikrat (s. Abschnitt 4.2) entspricht nicht unbedingt der Konstruktion solcher Gremien.
} 
angeordneten Aufgaben dadurch nicht beeinträchtigt wird (§ 23 (4)).

Die nicht gegebene Kompetenz bezüglich der Prioritätensetzung im Großen - Programmautonomie - ist in mancher Hinsicht und im Blick auf die Vision eines kohärenten statistischen Systems sicher zu bedauern. In der Praxis wiegt das Fehlen dieser Autonomie weniger schwer. Vor dem Hintergrund der Budgetrestriktionen werden die ProgrammPrioritäten (leider) schon längst nicht mehr auf nationaler Ebene, sondern durch Instanzen auf europäischer Ebene gesetzt.

Soll ein größerer Gestaltungsspielraum verfügbar werden, wird dieser durch gute Argumente erarbeitet werden müssen. Die politischen Entscheidungsträger werden zu überzeugen sein, warum zusätzliche österreich-spezifische Arbeiten notwendig sind, warum Projekte, welche aus dem Systemzusammenhang heraus gemacht werden sollten, unerläßlich sind.

\subsection{4 ,Verrechtlichung“6 der Statistik}

Generell gilt das Prinzip, daß keine Statistik ohne gesetzliche (nationale oder supranationale) Anordnung oder ohne Verordnung nach dem Bundesstatistikgesetz erstellt werden kann. Diese Verrechtlichung resultiert unmittelbar aus den Grundforderungen an das Gesetz,

- die Respondentenbelastung zu minimieren (der Fall der Primärstatistik),

- die rechtlichen Voraussetzungen nach dem Datenschutzgesetz zu schaffen (der Fall der Sekundärstatistik) und

- die für statistische Aufgaben erforderlichen öffentlichen Mittel möglichst gering zu halten (für beide Fälle).

Die Verrechtlichung spiegelt auch die Sicht wieder, daß die Beantwortung der Frage nach dem ,was“ - dem statistischen Programm - eine eminent politische, die nach dem „wie“ - den Verfahren, den Methoden - eine wissenschaftlich/fachliche Aufgabe ist.

Als Ausnahme von der generellen Regel können nach $\S 16$ (2) Befragungen auf der Grundlage der freiwilligen Auskunftserteilung ohne Anordnung durch Gesetz oder Verordnung durchgeführt werden, was ein Mindestmaß an Flexibilität sichert.

\subsubsection{Zugang zu personenbezogenen Daten, Registerabgleich natürliche Personen}

Im ursprünglichen, am 25. Jänner 1999 zur Begutachtung ausgesandten Entwurf war noch eine Regelung über eine „Statistische Kennummer“ enthalten: Die Einführung einer solchen zentralen Meldenummer in einem Zentralen Melderegister war für eine Novelle zum Meldegesetz 1991 für jede gemeldete Person geplant. Ihr hätte dann auch die Funktion zukommen können, als statistische Kennummer zu fungieren.

Die Idee hinter der Schaffung dieser Kennummer war, einen eindeutigen Zuordnungsbegriff für alle Verwaltungsdaten aufzubauen. Eine Identifikationskennzahl existiert de facto für viele Bereiche der Verwaltung schon jetzt in Form der Sozialversicherungsnummer (so z.B. für alle Belange der Sozialversicherung, der Finanzämter). Hierfür die 
Kennzahl eines Zentralen Melderegisters zu verwenden, wäre umfassender und leichter handhabbar gewesen.

Für Datenzusammenführungen hätte die Kennummer nur verwendet werden dürfen, wenn dafür die Voraussetzungen nach $\S 10$ (1) gegeben gewesen wären. Mit dieser Bestimmung hätte in Österreich eine Möglichkeit eröffnet werden sollen, für deren Nutzung es in Europa einige beeindruckende Beispiele gibt. Besonders in den skandinavischen Staaten kann die mustergültige Verwendung der Technik der Datenzusammenführung über Kennummern für statistische Zwecke studiert werden.

Der Datenschutzrat hat im Rahmen des Begutachtungsverfahrens die Zustimmung zu dieser geplanten Vorgangsweise versagt. Es wurde der Statistischen Kennzahl unterstellt, ein „Personenkennzeichen durch die Hintertür“ zu sein. Aus der Sicht einer effektiven, sparsamen und den Bürger wenig belastenden Bundesstatistik ist dies sehr zu bedauern.

Der in der Bundesstatistik in besonderem Ausmaß zu praktizierende Datenschutz und der Umstand, daß die Anstalt ,Statistik Österreich“ innerhalb der Verwaltung noch einmal einen extrem geschützten und abgeschotteten Bereich darstellt, den nur anonymisierte und aggregierte Informationen verlassen dürfen, lassen hoffen, daß hier in naher Zukunft eine neue Gesprächsbasis mit dem Datenschutzrat gefunden werden kann und gemeinsam eine akzeptable Lösung erdacht wird. Die äußerst positiven Beispiele aus dem Ausland könnten helfen, aufzuzeigen, wie groß einerseits die Einsparungspotentiale, andererseits die möglichen Qualitätsverbesserungen sind.

\subsubsection{Metakompetenz für Verwaltungs- und Registerinformation}

Als Voraussetzung für die effiziente Nutzung von Verwaltungsdaten gelang es in $\S 10$, umfassende Mitwirkungspflichten der registerführenden Stellen und von Institutionen, die über Verwaltungsdaten oder Statistikdaten verfügen, aufzunehmen. Wie schon erwähnt, haben sie ihre Daten nach Maßgabe einer Anordnung kostenlos und wenn möglich auf elektronischem Wege bereitzustellen, sie haben die so wichtigen Metainformationen offenzulegen und sie haben bei Änderungen auf die Bedürfnisse der statistischen Nutzung Rücksicht zu nehmen.

Nicht möglich war es - was man als Statistiker natürlich sehr wünschen würde - die Anstalt als „Oberbehörde“ zu installieren, die (um der Statistik willen) direkt Einfluß auf die Gestaltung aller Verwaltungsdaten nehmen könnte. Aufgabe der Leitung und des Statistikrates wird es sein, Verständnis und Kooperationsbereitschaft zu wecken.

\section{Wahl der Organisationsform - Umwandlung des Öster- reichischen Statistischen Zentralamts in eine Anstalt öffentlichen Rechts}

\subsection{Die Bundesanstalt „Statistik Österreich“ als Instrument zur Um- setzung der Vorgaben des ,Statistikteils“ des Gesetzes}

Mit der Umwandlung des Österreichischen Statistischen Zentralamts in eine Anstalt öffentlichen Rechts mit dem Namen „Statistik Österreich“ soll der Leitung im Sinne des „New 
Public Managements" mehr Flexibilität bei größerer Eigenverantwortlichkeit eingeräumt werden. Ohne die Amtliche Statistik zu privatisieren oder zu kommerzialisieren, sind bei der Führung privatwirtschaftliche Grundsätze verstärkt zu berücksichtigen. In vielen Bestimmungen orientieren sich die Vorgaben an den Grundsätzen des Aktienrechts.

Die zentrale Stellung der Amtlichen Statistik im Rahmen des gesamten statistischen Systems wird von der Wahl einer neuen Organisationsform nicht beeinträchtigt. Die Anstalt „Statistik Österreich“ wird weiterhin insbesondere alle internationalen Verpflichtungen wahrzunehmen haben, die von nationalen statistischen Einrichtungen zu erbringen sind. Die Bundesanstalt ist insbesondere für folgende Aktivitäten zuständig:

- Erstellung aller nach $\S 4$ angeordneten Statistiken.

Nach $\S 23$ (1)) sind dies Statistiken, die:

1. durch einen innerstaatlich unmittelbar wirksamen internationalen Rechtsakt,

2. durch Bundesgesetz oder

3. durch eine Verordnung gemäß Abs. 3 angeordnet sind.

- Internationale Vertretung Österreichs als Nationales Statistisches Institut $(\S 23(3,4,7))$

- Durchführung klassifikatorischer Zuordnungen (§ 23 (5))

- Führung zentraler Register für statistische Zwecke ( $\S 25$ und 26).

Darüber hinaus darf die Bundesanstalt als Auftragnehmer sonstige Statistiken auf Grund vertraglicher Vereinbarung für den Bund, die Länder, die Gemeinden und sonstige juristische Personen öffentlichen Rechts sowie für die Nationalbank erfüllen und für Organe der Europäischen Union und internationale Organisationen Arbeiten gegen Kostenersatz erstellen ( 23 (2)). Für andere Auftraggeber darf die Bundesanstalt nicht tätig werden.

Die Bundesanstalt ist andererseits nach $\S 27$ ermächtigt, als Auftraggeber zu fungieren. Durch Vertrag können Dritte mit der Durchführung von statistischen Erhebungen oder der Erstellung von Statistiken beauftragt werden, wenn dies ökonomisch sinnvoll ist und dem weder schutzwürdige Interessen der Betroffenen noch öffentliche Interessen entgegenstehen. Bei allen Gesetzes- und Verodnungsentwürfen, die Auswirkungen auf die Bundesstatistik haben können, ist der fachliche Rat der Bundesanstalt einzuholen ( $\S$ 13).

Die Anstalt wird von einem fachlichen und einem kaufmännischen Leiter, analog zu zwei Vorständen einer Kapitalgesellschaft, geführt ( $(38)$. Der fachliche Leiter ist für die Leitung der Bundesanstalt in fachlichen und hoheitlichen Aufgaben zuständig. Soweit er hoheitlich tätig ist, unterliegt er den Weisungen des zuständigen Bundesministers. In allen wissenschaftlich/methodischen Fragen ist er bei der Erfüllung der ihm obliegenden Aufgaben aber weisungsfrei. Dem kaufmännischen Geschäftsführer obliegt die betriebswirtschaftliche Leitung der Bundesanstalt. Dabei hat er die Sorgfaltspflichten eines ordentlichen Geschäftsmannes anzuwenden und die kaufmännischen Grundsätze zu beachten. 
Das Gesetz gibt der Leitung der Anstalt Flexibilität und mehr Planbarkeit zumindest für einige Jahre. Es erfüllt damit einen der immer wieder geäußerten Wünsche zur Verbesserung der Produktionsbedingungen der Statistik. Neben einem Jahrespauschalbetrag werden Aufwendungen für die Startphase ersetzt. Für EDV-Investitionen, für die Erfüllung der zusätzlichen Publikationsverpflichtungen und anderer zusätzlicher Aufgaben sowie für durch die neue Rechtsform bedingte Ausgaben werden bis zum Jahr 2002 insgesamt weitere Mittel von fast 100 Mio. S bereitgestellt.

Insgesamt gesehen schafft die gewählte Organisationsform - im Vergleich zu der Alternative Bundesdienststelle - bessere Voraussetzungen, um die Vorgaben des „Statistikteils" des Gesetzes umzusetzen. Zwei zentrale Kontroll- und Aufsichtsgremien sind vorgesehen:

\subsection{Der Statistikrat als Garant von Unabhängigkeit und Qualität}

Dem Statistikrat ist die Kontrolle in fachlich/wissenschaftlicher Hinsicht vorbehalten. Mit der Installierung des aus 15 Fachleuten zusammengesetzten Gremiums mit weitreichenden Kontrollkompetenzen soll gesichert werden, daß die hohen vorgegebenen Standards auch in der Praxis verwirklicht werden. Den verstärkten Bemühungen um Wissenschaftlichkeit entspricht, daß dem Statistikrat zumindest ein habilitierter Wissenschaftler angehören soll. Zu den Aufgaben des Statistikrates zählen auch die Bemühungen um eine institutionenübergreifende Koordination aller statistischer Aktivitäten.

Auch der Statistikrat verfügt über keine Metakompetenz. Er kann aber Empfehlungen aussprechen und Stellungnahmen abgeben. Vor allem aber hat er dem Bundeskanzler jährlich einmal einen Tätigkeitsbericht zu übermitteln, der von der Bundesregierung dem Nationalrat vorzulegen ist. Damit ist eine ausreichende Öffentlichkeit für seine Stellungnahmen gesichert.

\subsection{Der Wirtschaftsrat}

Der Wirtschaftsrat hat über die wirtschaftliche Führung der Anstalt zu wachen. Die Aufgaben des Wirtschaftsrats entsprechen weitestgehend jenen des Aufsichtsrats einer Aktiengesellschaft.

Über die Institutionen Statistikrat und Wirtschaftsrat soll eine Abstimmung der Ziele Reorientierung, Qualität und Wirtschaftlichkeit erreicht werden. Auf der operativen Ebene sind zwei Generaldirektoren, ein fachlicher und ein kaufmännischer, vorgesehen. Die Analogie zum Zusammenspiel zwischen einem technischen und einem kaufmännischen Vorstand in einem Produktionsbetrieb oder zu einem künstlerischen und einem kommerziellen Leiter in Kulturbetrieben (z.B. LIVA Linz) ist evident.

\section{An Stelle einer Zusammenfassung: Die Vision eines neuen Statistikpaktes mit den Bürgern}

Das Bundesstatistikgesetz 2000 zeigt, daß im Verhältnis der Bundesstatistik zum Bürger ein Umdenken eingesetzt hat. Der Bürger wird nicht mehr einseitig als Datenlieferant ge- 
sehen, dem der Staat jederzeit und in beliebiger Dichte Informationen abverlangen kann. Informationen, die der Staat schon hat, werden auch für die Statistik genutzt, Mehrfacherhebungen und somit Belastungen und Kosten sind zu vermeiden. Das Bundesstatistikgesetz garantiert mit einer Reihe von Regelungen, daß der Bürger so schonend wie nur möglich behandelt wird.

Die Bundesstatistik schließt mit dem Bürger einen Pakt. Er gibt der Bundesstatistik Information, die sie sonst nirgends beschaffen kann, die Statistik ermöglicht ihm dafür aber den Zugang zu allen Ergebnissen, sodaß er sich ein Bild über den Zustand unserer Gesellschaft machen kann. Man kann diesen Pakt als Handel sehen, aus dem beide Partner Gewinn ziehen. Beide wissen nachher mehr als vorher.

Statistik wird als öffentliches Gut gesehen. Die Konsequenz ist eine neue Informationspolitik. Alle Hauptergebnisse werden kostenlos im Internet angeboten, für Detailergebnisse oder Sonderauswertungen darf nur der zusätzliche Aufwand für die Erstellung verrechnet werden. Politik und Öffentlichkeit sind in gleichem Ausmaß und vor allem gleichzeitig zu informieren - es gibt keine Informationsprivilegien mehr.

Mit der „Statistik Österreich“ soll unserer Informationsgesellschaft ein neues Kompetenzzentrum geschaffen werden. Die neue Bundesanstalt wird nach den Grundsätzen des TQM (Total Quality Management) und im Sinne einer wirkungsorientierten Dienstleistung (NPM) zu führen sein. Ein Wirtschaftsrat wird einen effektiven und effizienten Mitteleinsatz überwachen. Ein Statistikrat garantiert für Qualität und Objektivität.

Die Weichen für ein neues Zeitalter der Bundesstatistik in Österreich sind gestellt.

\section{Literatur}

Beirat für Wirtschafts- und Sozialfragen. Überlegungen zur Lage der amtlichen Statistik in Österreich. Wien, 1996.

H. Brüngger. Was soll in einem modernen Statistikgesetz enthalten sein? In: Österreichisches Statistisches Zentralamt, Hrsg., Protokoll der Ordentlichen Jahresversammlung 1998 der Statistische Zentralkommission, S. 19-30. Wien, 1998.

Bundesstatistikgesetz 2000. Bundesgesetz über die Bundesstatistik, BGB1. Nr. I 163/1999.

Datenschutzgesetz 2000. Bundesgesetz über den Schutz personenbezogener Daten, BGB1. I $165 / 1999$.

W. De Vries. Die amtliche Statistik und der europäische Integrationsprozeß. In: Statistisches Bundesamt, Hrsg., Amtliche Statistik - Ein konstitutives Element des demokratischen Staats, S. 47-58. Wiesbaden, 1999.

W. De Vries, W. Keller, A. Willeboordse. Reducing the Response Burden: Some Developments in the Netherlands. International Statistical Review, 2:199-213, 1996.

C. Engelage. Rechtlicher und organisatorischer Status der amtlichen Statistik. In: R. Stäglin und G. Wagner, Hrsg., Auswirkungen gesellschaftlicher Veränderungen auf Funktion und Arbeitsweise der amtlichen Statistik - Berlin als Beispiel, S. 140-142. DIW Berlin, 1990. 
I. Fellegi. Characteristics of an Effective Statistical System. International Statistical Review, 2:165-197, 1996.

Y. Franchet. Funktion der Statistik bei der europäischen Integration. Allgemeines Statistisches Archiv, 1:18-25, 1995.

H. Grohmann. Auskunftspflicht versus Freiwilligkeit bei Erhebungen der amtlichen Statistik und einige Bemerkungen zu deren rechtlichem Status. In: R. Stäglin und G. Wagner, Hrsg., Auswirkungen gesellschaftlicher Veränderungen auf Funktion und Arbeitsweise der amtlichen Statistik - Berlin als Beispiel, S. 55-66. DIW Berlin, 1990.

P. Hackl. Wünsche an ein österreichisches Bundesstatistik-Gesetz. Österreichische Zeitschrift für Statistik, 2:71-84, 1997.

J. Hahlen. Amtliche Statistik als zentraler Teil der statistischen Infrastruktur. Anmerkungen zum Memorandum. Allgemeines Statistisches Archiv, 3-4:387-390, 1998.

W. Haug, u.a. Herausgeber. Statistik im Dienste der Öffentlichkeit; Statistik der Schweiz, Ordner Nr. 246-9700. SFSO Bern, 1998.

D. Hiss und W. Schröder. Amtliche Statistik - künftig unabhängig wie die Deutsche Bundesbank? In: R. Stäglin und G. Wagner, Hrsg., Auswirkungen gesellschaftlicher Veränderungen auf Funktion und Arbeitsweise der amtlichen Statistik - Berlin als Beispiel, S. 129-133. DIW Berlin, 1990.

C. Hohmann-Dennhardt. Amtliche Statistik und ihre Bedeutung für die demokratische Entwicklung der Bundesrepublik Deutschland. In: Statistisches Bundesamt, Hrsg., Amtliche Statistik - Ein konstitutives Element des demokratischen Staats, S. 15-23. Wiesbaden, 1999.

International Statistical Institute. Declaration on Professional Ethics. International Statistical Review, 227-242, 1986.

K. Klein. Das neue Bundesstatistikgesetz. Statistische Nachrichten, 8:628-632, 1999.

W. Klitsch. Bundesstatistik und „Schlanker Staat“. Allgemeines Statistisches Archiv, 340341, 1996.

H. Lützel. Registergestützte Datengewinnung - Überlegungen zur Umsetzung der EUUnternehmensstatistiken. In: Statistisches Bundesamt, Hrsg., Amtliche Statistik - Ein konstitutives Element des demokratischen Staats, S. 75-80. Wiesbaden, 1999.

Meldegesetz 1991. Bundesgesetz über das polizeiliche Meldewesen, BGB1. Nr. 9/1992.

J. Richter. Wirtschaftsstatistik und Europäische Integration - Einige Konsequenzen für die empirische Wirtschaftsforschung. Jahrbücher für Nationalökonomie und Statistik, 1:92-110, 1996.

R. Stäglin. Was bleibt von der nationalen Statistik nach Herstellung der Wirtschafts- und Währungsunion? Allgemeines Statistisches Archiv, 1:26-46, 1995. 
UN-ECE. The Fundamental Principles of Official Statistics in the Region of the Economic Commission for Europe. Genf, 1992.

Verordnung (EG) Nr. 322/97. Verordnung des Rates vom 17. Feber 1997 über die Gemeinschaftsstatistiken. Europäisches Statistikgesetz.

Verordnung (EG) Nr. 2533/98. Verordnung des Rates vom 23. November 1998 über die Erfassung statistischer Daten durch die Europäische Zentralbank. EZB StatistikVerordnung.

P. Von der Lippe. Privatisierung der amtlichen Statistik? Wirtschaft und Statistik, 11:928931, 1998.

P. Von der Lippe. Privatisierung der amtlichen Statistik? (Diskussionsbeitrag). Wirtschaft und Statistik, 8:660-665, 1999.

R. Wiegert. Zur Gegenwart und Zukunft der amtlichen Statistik in Deutschland. Jahrbücher für Nationalökonomie und Statistik, 1:138-150, 1998.

A. Willeboordse. Monitoring the response burden for business surveys. Netherlands Official Statistics, 5-10, Spring 1996.

H. Zeuthen. Die Organisation der amtlichen Statistik in Dänemark. In: R. Stäglin und G. Wagner, Hrsg., Auswirkungen gesellschaftlicher Veränderungen auf Funktion und Arbeitsweise der amtlichen Statistik - Berlin als Beispiel, S. 115-128. DIW Berlin, 1990.

Adresse der Autoren:

Hofrat Dr. Ewald Kutzenberger

Amt der Oberösterreichischen Landesregierung

Statistischer Dienst

Kärntnerstr. 16

A-4020 Linz, Österreich

Tel.: +430732 77203274

Fax: +430732 77203294

Email: Ewald.Kutzenberger@ooe.gv.at
Dr. Josef Richter

Wirtschaftskammer Österreich

Statistische Abteilung

Postfach 180

A-1045 Wien, Österreich

Tel.: +431501054114

Fax: +43150105246

Email: Richterj@statistik.wk.or.at 